\title{
Upper Bounds on the $D(\beta)$-Vertex-Distinguishing Edge-Chromatic Numbers of Graphs ${ }^{\star}$
}

\author{
Tian Jing-jing ${ }^{1,2}$, Liu Xin-sheng ${ }^{1}$, Zhang Zhong-fu ${ }^{1}$, and Deng Fang-an ${ }^{1}$ \\ ${ }^{1}$ Department of Mathematics, Shaanxi University of Technology, \\ Hanzhong, Shaanxi 723001, China \\ ${ }^{2}$ College of Mathematics and Information Science, Northwest Normal University, \\ Lanzhou, Gansu 730070, China \\ tianjj_1225@yahoo.com.cn
}

Abstract. In this paper, let $d$ be the maximum degree of
the upper bounds for the $D(\beta)$-vertex-distinguishing edg
number by probability method and prove that
\[ \chi_{\beta-v d}^{\prime}(G) \leq \begin{cases}2 \sqrt{2(\beta-1)} d^{\frac{\beta+2}{2}}, & d \geq 4, \beta \geq 4 ; \\ 8 d^{\frac{5}{2}}, & d \geq 6, \beta=3 ; \\ 32 d^{2}, & d \geq 4, \beta=2 .\end{cases} \]

It is well known to compute the chromatic number of a graph is NP-Hard in graph theory. In the past, the people have some results about it by combined methods, see [1, 2, 3].At ICM2002, Noga Alon advanced a new theory that graph coloring could studied by probability methods. For instance, some conclusions have been gotten by probability methods, see[4][5][6][7]. In 2006, Zhang Zhongfu present a new concept of the $\alpha$ - $D(\beta)$-vertex-distinguishing proper edge-coloring and conjecture, see [8]. In this paper, let $d$ be the maximum degree of $G$, we study the upper bounds for the $D(\beta)$-vertex-distinguishing edge-chromatic number by probability method

All the graphs $G=G(V, E)$ discussed in this paper are finite, undirected, simple and connected. Let $d$ be the maximum degree of $G$ and $\delta$ be the minimum degree of $G$.

Definition 1. $\alpha$ - $D(\beta)$-vertex-distinguishing proper edge-coloring $D(\beta)$-vertexdistinguishing edge-chromatic number, see [8].

Definition 2. dependency digraph, see [10].

Lemma 1 ((The General Local Lemma)). see[9][10]

Please refer to [11] for other terminologies and notations.

\footnotetext{
* Partial support was provided by the National Natural Science Foundation Grants of China (No. 40301037) and Natural Science Foundation of Gansu Province (3ZS051A25-025).
} 
Theorem 1. Let $d$ be the maximum degree of $G$, then

$$
\chi_{\beta-v d}^{\prime}(G) \leq \begin{cases}2 \sqrt{2(\beta-1)} d^{\frac{\beta+2}{2}}, & d \geq 4, \beta \geq 4 \\ 8 d^{\frac{5}{2}}, & d \geq 6, \beta=3 \\ 32 d^{2}, & d \geq 4, \beta=2 .\end{cases}
$$

Proof. We only give the proof for the case that $\beta \geq 4, d \geq 4$, the other cases can be proved similarly. The proof consists of five steps. We assign to each edge of $G$ a uniformly random coloring from $\left\{1,2, \ldots, 2 \sqrt{2(\beta-1)} d^{\frac{\beta+2}{2}}\right\}$ named this new coloring $f$. We will use Lemma 3 to show that a positive probability, $f$ is a $D(\beta)$-vertex-distinguishing proper edge-coloring.

Step 1. The following bad events are defined :

$<1>$ For each pair of incident edges $e, f$, let $A_{e, f}$ be the event that both $e$ and $f$ are colored with the same color;

$<2>$ For each edge $e=u w$, such that $\operatorname{deg}(u)=\operatorname{deg}(w) \geq \delta(G)$, let $B_{e}$ be the set of all edges which are connected $u$ or $w$, then $E_{B_{e}}$ is the events that the edges which are adjacent to $u$ and $w$ are colored properly, and $C(u)=C(w)$;

$<3>$ For each path whose length is $2, P_{u v}=u e w f v$, such that $\operatorname{deg}(u)=$ $\operatorname{deg}(v) \geq \delta(G)$, let $P_{u v}$ be the set of all edges which are connected $u$ or $v$, then $E_{P_{u v}}$ is the event that the paths which are adjacent to $u$ and $v$ are colored properly, and $C(u)=C(v)$;

$<\beta+1>$ For each path whose length is $\beta, P_{u(\beta)}=u e w f v \ldots, \beta$, such that $\operatorname{deg}(u)=\operatorname{deg}(\beta) \geq \delta(G)$ let $P_{u \beta}$ be the set of all edges which are connected $u$ or $\beta$, then $E_{P_{u \beta}}$ be the event that the edges which are adjacent to $u$ and $\beta$ are colored properly, and $C(u)=C(\beta)$.

It remains to show that with positive probability none of these events happen, then $f$ is a $D(\beta)$-VDPEC of $G$. Let us construct dependency graph $H$ whose nodes are all the events of two nodes $E_{X}$ and $E_{Y}$ are adjacent if and only if $X$ and $Y$ contain at least one common edge. Since the occurrence of each event $E_{X}$ depends only on the edges of $X, H$ is dependency graph for our events. In order to apply The General Local Lemma, we need estimates for the probability of each event and the number of nodes of each type in $H$ which are adjacent to any give node.These estimates are given in the two steps below.

Step 2. Estimate the probability of each event:

$$
\begin{aligned}
\operatorname{Pr}\left(A_{e, f}\right) & =\frac{1}{2 \sqrt{2(\beta-1)} d^{\frac{\beta+2}{2}}} ; \\
\operatorname{Pr}\left(E_{B_{e}}\right) & =\frac{1}{\left(\begin{array}{c}
2 \sqrt{2(\beta-1)} d^{\frac{\beta+2}{2}} \\
1
\end{array}\right)+\left(\begin{array}{c}
2 \sqrt{2(\beta-1)} d^{\frac{\beta+2}{2}} \\
2
\end{array}\right)+\ldots+\left(\begin{array}{c}
2 \sqrt{2(\beta-1)} d^{\frac{\beta+2}{2}} \\
d-1
\end{array}\right)} \\
& <\frac{1}{(d-1) 2 \sqrt{2(\beta-1)} d^{\frac{\beta+2}{2}}} ; \\
\operatorname{Pr}\left(E_{P_{u v}}\right) & =\operatorname{Pr}\left(E_{P_{u r}}\right)=\ldots=\operatorname{Pr}\left(E_{P_{u(\beta-1)}}\right)=\operatorname{Pr}\left(E_{P_{u \beta}}\right)
\end{aligned}
$$




$$
\begin{aligned}
& =\frac{1}{\left(2 \sqrt{2(\beta-1)} d^{\frac{\beta+2}{2}}\right)^{d}} \frac{1}{\left(2 \sqrt{2(\beta-1)} d^{\frac{\beta+2}{2}}\right)^{d}} \\
& =\frac{1}{\left(2 \sqrt{2(\beta-1)} d^{\frac{\beta+2}{2}}\right)^{2 d}} .
\end{aligned}
$$

\begin{tabular}{|c|c|c|c|c|c|c|}
\hline event & $\langle 1\rangle$ & $\langle 2\rangle$ & $\langle 3\rangle$ & $\langle 4\rangle$ & $\cdots$ & $\langle\beta+1\rangle$ \\
\hline$A_{e, f}$ & $4 d-5$ & $3 d-2$ & $\begin{array}{c}2(d-1)^{2}+ \\
d(d-1)\end{array}$ & $\begin{array}{c}2(d-1)^{3} \\
+d(d-1)^{2}\end{array}$ & & $\begin{array}{c}2(d-1)^{\beta} \\
+d(d-1)^{\beta-1}\end{array}$ \\
\hline$E_{B_{e}}$ & $(2 d-1)(2 d-2)$ & $\begin{array}{c}(2 d-2) d \\
+1\end{array}$ & $(2 d-1) d(d-1)$ & $(2 d-1) d(d-1)^{2}$ & & $(2 d-1) d(d-1)^{\beta-1}$ \\
\hline$E_{P u v}$ & $\begin{array}{c}2 d(2 d-1) \\
+1+2(d-2)\end{array}$ & $\begin{array}{c}2 d^{2} \\
+d-2\end{array}$ & $\begin{array}{l}2(d-1)^{3} \\
+d(d-1)\end{array}$ & $2(d-1)^{4}+d(d-1)^{2}$ & & $\begin{array}{c}2(d-1)^{\beta+1} \\
+d(d-1)^{\beta-1}\end{array}$ \\
\hline$E_{P u r}$ & $\begin{array}{c}2(d-1)(2 d-1) \\
+2+4(d-2)\end{array}$ & $\begin{array}{c}2 d^{2}+2 d \\
-3\end{array}$ & $\begin{array}{c}2 d(d-1)^{2} \\
+2 d(d-1)-3\end{array}$ & $\begin{array}{c}2 d(d-1)^{3} \\
+2 d(d-1)^{2}-3\end{array}$ & & $\begin{array}{c}2 d(d-1)^{\beta}+ \\
2 d(d-1)^{\beta-1}-3 \\
\end{array}$ \\
\hline$\ldots$ & $\begin{array}{c}\ldots \\
\end{array}$ & $\ldots$ & $\ldots$ & $\ldots$ & $\ldots$ & $\ldots$ \\
\hline$E_{P_{u \beta}}$ & $\begin{array}{c}2(d-1)(2 d-1) \\
+(\beta-1)+ \\
2(\beta-1)(d-2)\end{array}$ & $\begin{array}{c}2 d^{2} \\
+(\beta-1) d \\
-\beta\end{array}$ & $\begin{array}{c}2 d(d-1)^{2}+ \\
(\beta-1) d(d-1)-\beta\end{array}$ & $\begin{array}{c}2 d(d-1)^{3}+ \\
(\beta-1) d(d-1)^{2}-\beta\end{array}$ & & $\begin{array}{c}2 d(d-1)^{\beta}+ \\
+\beta-1 \\
(\beta-1) d(d-1)^{\beta-1}-\beta\end{array}$ \\
\hline
\end{tabular}

Step 3. Estimate the dependency events number, in the following table:

Step 4. Find the real constant $x_{i}\left(0 \leq x_{i} \leq 1\right)$ for applying Lemma 3 . Let $\sqrt{2(\beta-1)} d^{\frac{\beta+2}{2}},(d-1) \sqrt{2(\beta-1)} d^{\frac{\beta+2}{2}},\left(\sqrt{2(\beta-1)} d^{\frac{\beta+2}{2}}\right)^{2 d}, \ldots,\left(\sqrt{2(\beta-1)} d^{\frac{\beta+2}{2}}\right)^{2 d}$ be the constants associated with events of Type $<1>,<2>, \ldots,<\beta+1>$.

Step 5. Conclude that with positive probability no events of Type $<1>,<$ $2>,<3>, \ldots,<\beta+1>$ provided that:

Let $m=\sqrt{2(\beta-1)} d^{\frac{\beta+2}{2}}$.

(1)

$$
\begin{aligned}
\frac{1}{2 m} \leq & \frac{1}{m}\left(1-\frac{1}{m}\right)^{4 d-5}\left(1-\frac{1}{(d-1) m}\right)^{3 d-2} \cdot\left(1-\frac{1}{m^{2 d}}\right)^{d(d-1)+2(d-1)^{2}} \\
& \cdot\left(1-\frac{1}{m^{2 d}}\right)^{2(d-1)^{3}+d(d-1)^{2}} \cdot \ldots \cdot\left(1-\frac{1}{m^{2 d}}\right)^{d(d-1)^{\beta-1}+2(d-1)^{\beta}} ;
\end{aligned}
$$

$$
\begin{array}{r}
\frac{1}{2(d-1) m} \leq \frac{1}{(d-1) m}\left(1-\frac{1}{m}\right)^{(2 d-1)(2 d-2)} \cdot\left(1-\frac{1}{(d-1) m}\right)^{(2 d-2) d+1} \\
\cdot\left(1-\frac{1}{m^{2 d}}\right)^{(2 d-1) d(d-1)} \cdot\left(1-\frac{1}{m^{2 d}}\right)^{(2 d-1) d(d-1)^{2}} \cdot \ldots \cdot\left(1-\frac{1}{m^{2 d}}\right)^{d(d-1)^{\beta-1}(2 d-1)} ;
\end{array}
$$


(3)

$$
\begin{array}{r}
\frac{1}{(2 m)^{2 d}} \leq \frac{1}{m^{2 d}}\left(1-\frac{1}{m}\right)^{2 d(2 d-1)+2(d-2)+1} \cdot\left(1-\frac{1}{(d-1) m}\right)^{2 d^{2}+d-2} \\
\cdot\left(1-\frac{1}{m^{2 d}}\right)^{2(d-1)^{3}+d(d-1)} \cdot\left(1-\frac{1}{m^{2 d}}\right)^{2(d-1)^{4}+d(d-1)^{2}} \\
\ldots \cdot\left(1-\frac{1}{m^{2 d}}\right)^{2(d-1)^{\beta+1}+d(d-1)^{\beta-1}}
\end{array}
$$

$(4-\beta+1)$

$$
\begin{array}{r}
\frac{1}{(2 m)^{2 d}} \leq \frac{1}{m^{2 d}}\left(1-\frac{1}{m}\right)^{2(d-1)(2 d-1)+(t-1)+2(t-1)(d-2)} \cdot\left(1-\frac{1}{(d-1) m}\right)^{2 d^{2}+(t-1) d-t} \\
\cdot\left(1-\frac{1}{m^{2 d}}\right)^{2 d(d-1)^{2}+(t-1) d(d-1)-t} \cdot\left(1-\frac{1}{m^{2 d}}\right)^{2 d(d-1)^{3}+(t-1) d(d-1)^{2}-t} \\
\cdot \ldots \cdot\left(1-\frac{1}{m^{2 d}}\right)^{2 d(d-1)^{\beta}+(t-1) d(d-1)^{\beta-1}-t} \cdot(t=3, \ldots, \beta)
\end{array}
$$

Now, since $\left(1-\frac{1}{z}\right)^{z} \geq \frac{1}{4}$ for all real $z \geq 2$, and $\left(\sqrt{2(\beta-1)} d^{\frac{\beta+2}{2}}\right)^{2 d} \geq\left(\sqrt{2(\beta-1)} d^{\frac{\beta+2}{2}}\right)^{2}$, we can prove that the inequalities $(1),(2), \ldots,(\beta+1)$ are true, when $d \geq 4, \beta \geq 4$, Using Lemma $3, G$ has $\left(2 \sqrt{2(\beta-1)} d^{\frac{\beta+2}{2}}\right)-D(\beta)$-VDPEC, when $d \geq 4, \beta \geq 4$.

This completes the proof.

\section{References}

1. Zhang Zhongfu, Liu Linzhong, Wang weifan, Adjacent strong Edge Coloring of Graphs, Applied Mathematics Letters, 5(2002) 623-626.

2. Wang Weifan, Equitable total coloring of graphs with maximum degree 3, Graphs and Combinatorics, 18(2002) 677-85.

3. Bazgan C., Harkat-Benhamdine A., Li Hao and et al, On the Vertex-distinguishing Proper Edge-colorings of Graphs, J. Combin Theory(Ser. B), 75(1999) 288-301.

4. Alon N, Sadakov B, Zaks, A. Acyclic Edge Coloring of Graphs, Journal of Graph Theory, 37(2001)157-167.

5. Sun yirong, Yan Jingzhi, Relationship Between Girth and Acyclic Edge Chromatic Numbet of Graphs, Journal of Mathematical Study Series B, 95(2005)246-256.

6. H. Hatami, $\Delta+300$ is a bound on the adjacent vertex distinguishing edge Chromatic Numbet of Graphs, Journal of Combinatorial Theory, 36(2)(2003)135-139.

7. Liu Xinsheng, An Mingqiang, Chen Xiang'en, An Upper Bound for the Adjacent Vertex-distinguishing Acyclic Edge Chromatic Number, sumbitted.

8. Zhang Zhongfu, Li Jinwen, $D(\beta)$-Vertex edge coloring of graph, Journal of Mathematical, 49(3)(2006) 703-708.

9. M. Michael, R. Bruce, Graph Coloring and the Probabilistic Method, Springer, 2002.

10. A. N. Spencer, J. H. Erdos, Paul, The Probabilistic Method, 1992.

11. J. A. Bondy, U. S. R. Marty, Graph Theory with Applications, The Macmillan Press Ltd, New York, 1976. 